Daniel Ruiz Prieto $0^{1, *}$

Francisco Pedro García Fernández²

1. Graduado en enfermería. Universidad de Jaén. Jaén. España.

2. Enfermero. Doctor por la Universidad de Jaén. Profesor del Departamento de Enfermería. Vicedecano de la Facultad de Ciencias de la Salud. Universidad de Jaén. Miembro del Comité Director del GNEAUPP. Jaén. España.

${ }^{*}$ Autor para correspondencia.

Correo electrónico: danielruizprieto@hotmail.com (Daniel Ruiz Prieto).

Recibido el 29 de noviembre de 2019; aceptado el 20 de enero de 2020.

\section{Uso de antisépticos y antibióticos en el manejo de la carga bacteriana de heridas crónicas}

\author{
Use of antiseptics and \\ antibiotics in the management \\ of the bacterial load of chronic \\ wounds
}

\section{RESUMEN}

Objetivo: El objetivo de esta revisión es analizar la eficacia de diferentes apósitos antisépticos y antibióticos locales tópicos en heridas crónicas infectadas o con signos de colonización crítica. Metodología: Para la elaboración de esta revisión exploratoria se han empleado varias bases de datos (PubMed, CINAHL, Scopus, Cuiden, Cochrane y LILACS), utilizando cadenas de búsqueda adaptadas a cada base. Se han incluido diferentes tipos de estudios originales y revisiones sobre la cicatrización, la curación completa y/o el coste-beneficio, realizando una estimación de los costes de los tratamientos según la disponibilidad de datos. Se han excluido estudios sobre quemaduras, literatura gris, casos clínicos o series de casos. Resultados: Se han seleccionado 20 artículos, de los cuales 17 son estudios originales y 3 son revisiones; 19 artículos tratan sobre apósitos antisépticos y 3 sobre antibióticos locales tópicos utilizados en heridas crónicas infectadas o con signos de colonización crítica, obteniéndose datos sobre el efecto que producen en la cicatrización, la curación completa y/o el costebeneficio. Todos los productos hallados tienen buenos resultados, aunque el más utilizado y eficaz en estas heridas es la plata. Conclusiones: La plata es el producto más eficaz para la curación completa o la disminución del tamańo; resulta muy rentable, ya que los costes de los tratamientos con plata son menores y se obtienen mayores beneficios para el paciente. El cloruro de diaquilcarbamilo es una buena alternativa, debido a que es un producto muy eficaz y rentable.

PALABRAS CLAVE: Apósitos, antisépticos, antibióticos, heridas crónicas.

\section{ABSTRACT}

Objective: The objective of this review is to analyze the efficacy of different antiseptic dressings and local topical antibiotics in infected chronic wounds or with signs of critical colonization. Methodology: For the elaboration of this exploratory review several databases have been used (PubMed, CINAHL, Scopus, Cuiden, Cochrane and LILACS), using search chains adapted to each base. Different types of original studies and reviews on healing, complete healing and/or cost-benefit have been included, making an estimate of the costs of the treatments according to the availability of data. Studies on burns, gray literature, clinical cases or cases series have been excluded. Results: 20 articles have been included, of which 17 are original studies and 3 are reviews, obtaining that 19 articles treat on antiseptic dressings and 3 on local topical antibiotics used in infected chronic wounds or with signs of critical colonization, obtaining data on the effect that they produce in healing, complete healing and/ or cost-benefit. All the products found have good results, although the most used and effective in these wounds is silver. Conclusions: Silver is the most effective product for the complete healing or reducing size, being very profitable, since the costs of silver treatments are lower, obtaining greater benefits for the patient. The dialkylcarbamoyl chloride is a good alternative, because it is a very effective and profitable product.

KEYWORDS: Dressings, antiseptics, antibiotics, chronic wounds.

\section{У INTRODUCCIÓN}

Las heridas crónicas son lesiones que se producen en la piel y que no finalizan el proceso de cicatrización en un periodo inferior a 6 semanas ${ }^{1}$, alargándose principalmente la fase inflamatoria ${ }^{2}$ y cicatrizando por segunda intención ${ }^{1}$. En algunas ocasiones existe un incremento de exudado, una agudización del dolor, mal olor y/o coloración ${ }^{3}$.

Los dos principales tipos de heridas crónicas existentes son las lesiones relacionadas con la dependencia (que incluyen las úlceras por presión y/o cizalla, las lesiones por roce-fricción y las lesiones cutáneas asociadas a la humedad ${ }^{4}$ ) y las úlceras de la extremidad inferior, entre las que se encuentran las úlceras venosas, arteriales o isquémicas, hipertensivas y neuropáticas ${ }^{2}$, entre otras.

El proceso de cicatrización consta de varias fases: a) la fase de hemostasia, en la que las plaquetas producen un tapón de hemostasia, b) la fase inflamatoria, donde los macrófagos fagocitan produciendo desbridamiento autolítico ${ }^{6}$ y limpieza de la herida de microorganismos y células muertas $\left.{ }^{5}, c\right)$ la fase proliferativa o de granulación, produciéndose la angiogénesis y la producción de una matriz de colágeno ${ }^{5}$, d) la fase de epitelización, en la que las células epiteliales migran por la lesión ${ }^{6}$ y e) la fase de remodelación o de contracción, en la que se forma el tejido cicatricial ${ }^{6}$, pero este proceso puede verse interrumpi- 
do por múltiples causas, entre las que destaca el aumento de la carga bacteriana de la herida.

Para el correcto manejo de la carga bacteriana es fundamental diferenciar el concepto de antibiótico y antiséptico. Un antibiótico es una sustancia con capacidad selectiva ${ }^{7,8}$, ya que puede destruir bacterias (bactericida) o inhibir su desarrollo (bacteriostático) ${ }^{7}$, mientras que un antiséptico no tiene capacidad de selección de los microorganismos sobre los que actúa ${ }^{8}$.

Son varios los tipos de apósitos antisépticos que podemos encontrar en el mercado; entre ellos están: la plata, el cadexómero yodado, la polihexanida de biguanida (PHMB), la miel ${ }^{79,10}$ y el cloruro de diaquilcarbamilo (DACC) $)^{9,10}$.

Por su parte, los antibióticos locales tópicos más utilizados son: la sulfadiazina de plata o argéntica $(\mathrm{SAg})^{7,10,11}$, el ácido fusídico ${ }^{7}$, la mupirocina $^{7,10}$ y la nitrofurazona ${ }^{11}$.

Cabe destacar que el coste del tratamiento se ve incrementado al aumentar la gravedad de la lesión, ya que aumenta el tiempo de curación y la cantidad de complicaciones, siendo la infección una de ellas. En Espańa, el coste anual destinado al tratamiento de úlceras por presión es de 461 millones de euros ${ }^{12}$.

Por todo ello nos planteamos los siguientes objetivos.

\section{Y OBJETIVOS}

- Comparar el efecto de diferentes apósitos antisépticos y antibióticos locales tópicos en la curación y/o cicatrización de heridas crónicas infectadas o con signos de colonización crítica.

- Determinar el coste-beneficio del uso de apósitos antisépticos y antibióticos locales tópicos en heridas crónicas infectadas o con signos de colonización crítica.

\section{У METODOLOGÍA}

\section{Tipo de estudio}

Se ha realizado una revisión exploratoria de la literatura científica sobre los diferentes apósitos antisépticos y antibióticos locales tópicos usados en el manejo de la carga bacteriana para determinar sus diferentes efectos en la curación, la cicatrización y/o el coste-beneficio en heridas crónicas infectadas o con signos de colonización crítica.

\section{Bases de datos consultadas}

En la tabla 1 se muestran las bases de datos que han sido consultadas, sus cadenas de búsqueda y el periodo de análisis de la bibliografía.

\section{Criterios de búsqueda}

Para la elaboración de esta revisión, la búsqueda de la documentación se inició en noviembre de 2018 y finalizó a mediados de enero de 2019, utilizándose varias palabras clave, como son: apósitos, antisépticos, antibióticos y heridas crónicas y sus equivalentes en inglés.

\section{Criterios de inclusión y exclusión de los artículos}

\section{Criterios de inclusión:}

C1: Tipos de estudios: revisiones sistemáticas o bibliográficas y artículos originales (ensayos clínicos, estudios retrospectivos y prospectivos, etc.).

C2: Artículos que relacionen el uso de apósitos antisépticos y/o antibióticos locales tópicos con la curación, la cicatrización y/o el coste-beneficio en heridas crónicas infectadas o con signos de colonización crítica en pacientes de cualquier sexo o edad.

C3: Artículos en español, portugués, inglés y francés.

C4: Artículos realizados en humanos.

\section{Criterios de exclusión:}

Ci: Cartas al director, artículos de opinión, editoriales y otra literatura gris. Cii: Estudios sobre el manejo de la carga bacteriana en quemaduras.

Ciii: Estudios sobre casos clínicos o series de casos.

\section{Análisis de la calidad metodológica}

Se ha realizado una evaluación de la calidad metodológica de los estudios que se incluyeron en la muestra final mediante la versión castellana de la herramienta Critical Appraisal Skills Programme (CASPe), utilizando diferentes plantillas según el tipo de estudio.

Se han usado las plantillas de ensayos clínicos ${ }^{13}$, revisiones ${ }^{14}$, estudios de cohortes $^{15} \mathrm{y}$ de evaluación económica ${ }^{16}$, incluyéndose en esta revisión aquellos artículos que han obtenido una puntuación mayor o igual a 5 puntos, de un total de 11 preguntas para los ensayos, los estudios de cohortes o las evaluaciones económicas, y mayor o igual a 5 puntos sobre 10 preguntas para las revisiones.

Para los estudios descriptivos prospectivos se ha utilizado la plantilla de cohortes ${ }^{15}$ adaptada con un total de 8 preguntas, incluyéndose aquellos que obtienen una puntuación igual o superior a 4 puntos. Las puntuaciones de cada artículo se pueden consultar en la tabla 2.

\section{Datos a considerar en el estudio}

Los datos de los artículos analizados que han sido de utilidad son: autor, ańo, título, país, tipo de estudio, objetivo, producto utilizado, resulta-

Tabla 1. Bases de datos consultadas

\begin{tabular}{|c|c|c|}
\hline Base de datos & \multicolumn{1}{c|}{ Cadena de búsqueda } & Periodo de búsqueda \\
\hline PubMed & (anti-infective dressing OR local antibacterial agents) AND chronic wound & $1962-2019+$ humans \\
\hline CINAHL & (antimicrobial dressing OR local anti-bacterial agents) AND chronic wound & $1982-2019+$ humans \\
\hline Scopus & (anti-infective dressing OR local anti-bacterial agents) AND chronic wound & $1996-2019$ \\
\hline Cuiden & (antisepticos OR antibacterianos) AND heridas cronicas & $1978-2019$ \\
\hline Cochrane & (anti-infective dressing OR local antibacterial agents) AND chronic wound & $1993-2019$ \\
\hline LILACS & (apositos antisepticos OR antibacterianos) AND heridas cronicas & $1982-2019+$ humanos \\
\hline
\end{tabular}


Tabla 2. Puntuaciones de los artículos (CASPe)

\begin{tabular}{|c|c|}
\hline Estudio & Puntuación CASPe \\
\hline Wang J et al., 2007'17 & $4 / 11$ \\
\hline Zölb C et al., 2016'18 & $4 / 11$ \\
\hline Karlsmark T et al., $2003^{19}$ & $6 / 11$ \\
\hline Meaume S et al., 2005²0 & $6 / 11$ \\
\hline Münter KC et al., 2006²1 & $7 / 11$ \\
\hline Kammerlander G et al., 2008²2 & $5 / 8$ \\
\hline Miller CN et al., $2010^{23}$ & $8 / 11$ \\
\hline Rueda López J et al., $2010^{24}$ & $4 / 8$ \\
\hline Sibbald RG et al., 201125 & $8 / 11$ \\
\hline Lenselink E et al., $2011^{26}$ & $6 / 11$ \\
\hline Manizate F et al., 201227 & $6 / 11$ \\
\hline Woo KY et al., $2012^{28}$ & $7 / 11$ \\
\hline 0’Meara S et al., $2014^{29}$ & $9 / 10$ \\
\hline Forlee M et al., 2014 & $5 / 8$ \\
\hline Mosti G et al., $2015^{31}$ & $8 / 11$ \\
\hline Krasowski G et al., 2015² & $7 / 11$ \\
\hline Nherera LM et al., $2016^{33}$ & $10 / 11$ \\
\hline Palumbo FP et al., $2016^{34}$ & $6 / 11$ \\
\hline Harding KG et al., $2016^{35}$ & $6 / 11$ \\
\hline Diaz R et al., $2017^{36}$ & $6 / 11$ \\
\hline Totty JP et al., $2017^{37}$ & $6 / 10$ \\
\hline Sibbald RG et al., $2017^{38}$ & $8 / 10$ \\
\hline
\end{tabular}

CASPe: Critical Appraisal Skills Programme

Fuente: elaboración propia.

dos generales medidos (cicatrización, curación completa y/o coste-beneficio), muestra, tipo de herida, intervención realizada y resultados en los pacientes.

\section{Método de agregación de datos}

Se ha realizado una agregación narrativa, debido a que con los datos obtenidos no es posible realizar una agregación matemática.

\section{Aspectos éticos}

No existen conflictos de interés por parte de los autores en la elaboración de esta revisión.

\section{Cálculo de costes}

Se ha realizado una estimación de los costes de los tratamientos de forma general o del coste por paciente, según disponibilidad de datos, tomando como referencia los precios por apósito.

Para ello, se ha calculado el número de apósitos por paciente multiplicado por el coste de cada apósito, o si se indica, el número total de apósitos usados multiplicado por el coste de cada uno; por tanto, el coste estimado es el coste material:

- Contreet Ag: no se ha calculado debido a que ya no se comercializa.

- Cutimed Sorbact: 2,44€/unidad.
- Aquacel Ag: 3,73€/unidad.

- Durafiber Ag: 9,46€/unidad.

- Atrauman Ag: 3,88€/unidad.

- Membrana multifuncional Bio-film: se ha calculado con el precio del apósito de miel que se comercializa en Espańa: L-Mesitran: 4,37€/ unidad.

\section{$\searrow$ RESULTADOS}

\section{Resultados de la búsqueda}

Los resultados de la búsqueda se detallan en la figura 1 .

De los 685 artículos que se han identificado tras la realización de la búsqueda, 101 han sido eliminados al estar duplicados entre las diferentes bases de datos.

De los artículos restantes, 517 han sido eliminados tras leer título y resumen, de los cuales 84 son en otro idioma: 57 en alemán, 2 en checo, 3 en chino, 4 en croata, 1 en holandés, 3 en italiano, 2 en japonés, 1 en rumano, 8 en ruso y 3 en ucraniano. Los otros 3 han sido eliminados por referirse exclusivamente a quemaduras.

De los artículos que se han analizado a texto completo, 45 han sido eliminados al no cumplir los criterios de inclusión/exclusión: 23 por no relacionar el uso de apósitos antisépticos y/o antibióticos locales tópicos con la curación, la cicatrización y/o el coste-beneficio en heridas crónicas infectadas o con signos de colonización crítica en pacientes de cualquier sexo o edad; 11 por ser estudios que no han sido realizados en seres humanos; otros 4 por tratarse de literatura gris, y 7 por ser casos clínicos o series de casos, los cuales tienen baja evidencia clínica.

El artículo de Wang et al. ${ }^{17}$ y el de Zölb et al. ${ }^{18}$ han sido eliminados por tener una baja calidad metodológica, ya que ambos obtuvieron una puntuación de 4 sobre 11 al evaluarlos con la herramienta CASPe ${ }^{15}$.

Finalmente se han incluido en esta revisión 20 artículos, recolectando datos sobre el uso de apósitos antisépticos y/o antibióticos locales tópicos y sus efectos sobre la cicatrización, la curación completa y/o el coste-beneficio obtenido.

\section{Resultados generales de los estudios}

En la tabla 3 se muestran las características generales de los estudios incluidos en la revisión. Los artículos datan desde $2003^{19}$ a $2017^{36-38}$ y han sido realizados en países de cuatro continentes. En Europa se realizaron 13, concentrando la mayor parte y distribuyéndose de la siguiente forma: Dinamarca ${ }^{19,21}$, Francia ${ }^{20}$, Suiza ${ }^{22}$, España ${ }^{24}$, Países Bajos $^{26}$, Reino Unido ${ }^{29,33,35,37}$, Italia ${ }^{31,34}$ y Polonia ${ }^{32}$. En América se han realizado 5 estudios: Canadá25,28,38, Estados Unidos ${ }^{27}$ y Guatemala ${ }^{36}$. En Oceanía se ha realizado 1 estudio, concretamente en Australia ${ }^{23}$. Por último, 1 estudio ha sido realizado en África, concretamente en Sudáfrica ${ }^{30}$.

Estos artículos tratan de un total de 7 productos diferentes como tratamiento para la curación y/o disminución del tamaño de este tipo de heridas y que se clasifican en: $a$ ) antisépticos (plata ${ }^{19-21,23,24,27-32,35,38}$, cadexómero yodado ${ }^{23,29,33,38}$, polihexanida de biguanida ${ }^{25,26,38}$, miel ${ }^{29,36,38}$ y cloruro de diaquilcarbamilo ${ }^{22,31,37}$ ) y $b$ ) antibióticos (sulfadiazina de plata $^{29,34,38}$ y mupirocina ${ }^{29}$ ).

De los 20 estudios incluidos en la revisión, 17 son estudios originales y 3 son revisiones, con un total de 19 artículos que tratan sobre apósitos antisépticos y 3 sobre antibióticos locales tópicos. De ellos, 18 analizan los efectos sobre la cicatrización, 15 sobre la curación completa y 11 sobre el coste-beneficio. 


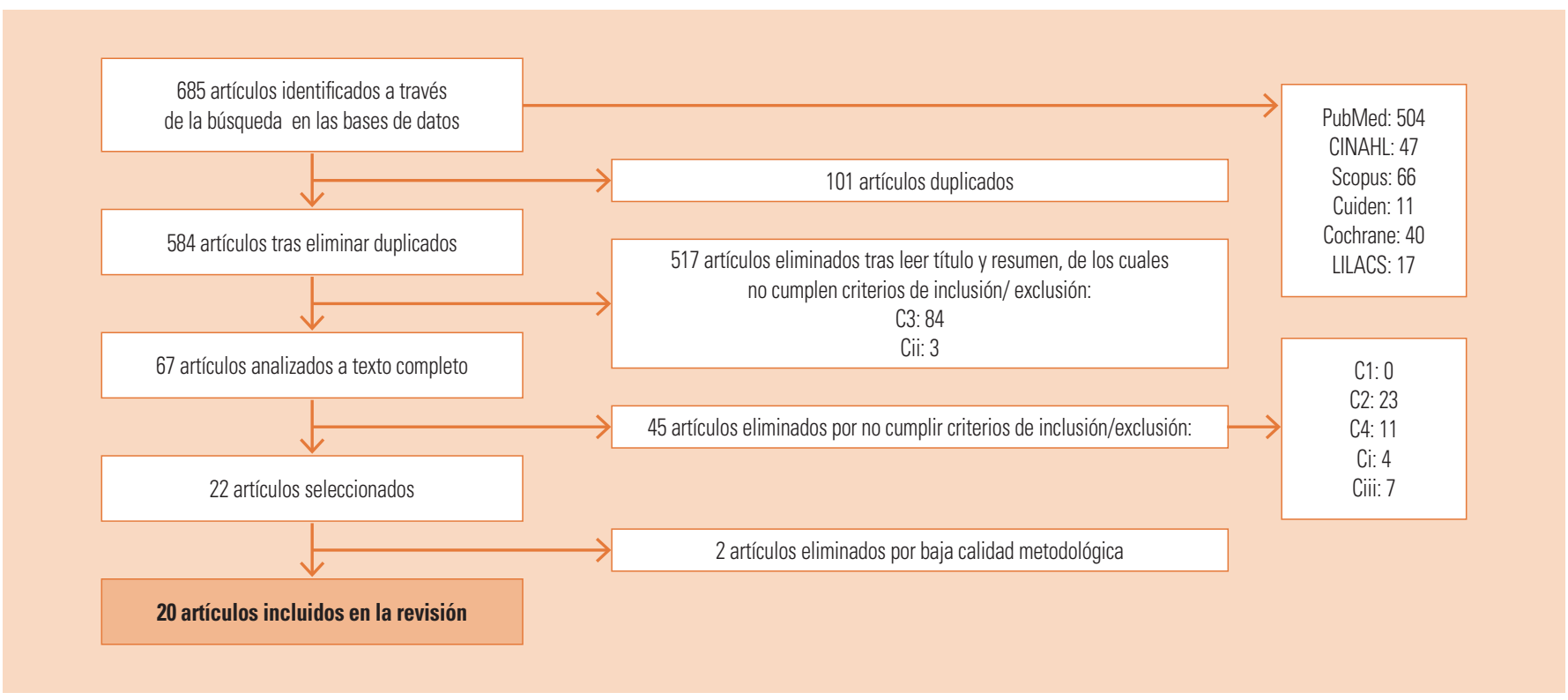

Figura 1. Diagrama de flujo de los estudios hallados tras la revisión.

Fuente: elaboración propia.

\section{Resultados sobre el efecto en la curación y/o cicatrización}

\section{UTILIZACIÓN DE LA PLATA}

La mayoría de los artículos ${ }^{19-21,23,24,28-30,32,35,38}$ muestran resultados positivos en la cicatrización o disminución del tamaño de las heridas al usar la plata.

En varios de ellos ${ }^{20,21,23,28,29,32,38}$ se realiza una comparación de la plata con otros productos. Los resultados son favorables en todos por parte de la plata frente al producto con el que se compara, excepto cuando se hace frente a la octenidina ${ }^{32}$, donde la plata obtiene peores resultados, $y$ frente a la sulfadiazina de plata, donde existe controversia ${ }^{38}$.

Las comparaciones que se realizan son con el alginato cálcico ${ }^{20}$, con las mejores prácticas locales ${ }^{21}$, con el cadexómero yodado ${ }^{23}$, con otros apósitos no antimicrobianos ${ }^{28,29}$, con la octenidina ${ }^{32}$, con placebo y con la sulfadiazina de plata ${ }^{38}$.

Sin embargo, en un artículo ${ }^{27}$ se compara la tasa de cierre con el uso de apósitos de colágeno bovino y plata frente a los de carboximetilcelulosa y plata, pero los resultados son no significativos.

Varios artículos ${ }^{19,20,24,30,32,35}$ muestran curación completa de heridas con el uso de la plata, mostrando mejores resultados que cuando se compara con otros productos como el alginato cálcico ${ }^{20}$, aunque en otros estudios los resultados al comparar con otros productos son no significativos, como con el cadexómero yodado ${ }^{23,29}$ o con apósitos no antimicrobianos $^{29}$.

Por último, al realizar una comparación de productos de plata entre sí y su efecto sobre la curación, también se obtienen otros datos que son no significativos, como los del colágeno bovino y plata frente a la carboximetilcelulosa y plata ${ }^{27}$ o los de un poliuretano de plata frente a la plata nanocristalina ${ }^{29}$.

\section{UTILIZACIÓN DEL CADEXÓMERO YODADO}

Son varios los artículos ${ }^{29,33,38}$ que comparan la tasa de curación de este producto frente a la atención estándar, obteniendo una tasa más favorable con el uso del cadexómero yodado.
También se compara el efecto en la cicatrización de este producto frente a la plata ${ }^{23}$, obteniendo el cadexómero yodado peores resultados.

En este mismo estudio ${ }^{23}$ y en otro ${ }^{29}$ los resultados en la curación al comparar el cadexómero yodado con la plata o con los apósitos hidrocoloides ${ }^{29}$ son no significativos.

\section{UTILIZACIÓN DE LA MIEL}

Sobre el uso de la miel se han obtenido resultados favorables al compararla con otros productos, consiguiendo una mayor cantidad de heridas curadas o que estaban cicatrizando, que al usar las curas tradicionales de húmedas a secas ${ }^{36}$.

Al comparar la disminución del tamańo que se produce al utilizar la miel frente a la povidona yodada se obtienen resultados similares ${ }^{38}$, aunque cuando comparamos el uso de la miel con el cuidado habitual ${ }^{29} \mathrm{o}$ la atención estándar ${ }^{38}$, el tiempo de curación es no significativo.

\section{UTILIZACIÓN DE LA POLIHEXANIDA DE BIGUANIDA}

El uso de la polihexanida de biguanida potencia la curación de las heridas ${ }^{26,38}$ y produce una notable disminución de su tamaño ${ }^{26}$.

Sin embargo, en otro artículo 25 , aunque los datos muestran mayor beneficio en la cicatrización con la polihexanida de biguanida frente a los apósitos de espuma no antimicrobianos, no pueden ser considerados, ya que son no significativos.

\section{UTILIZACIÓN DEL CLORURO DE DIAQUILCARBAMILO}

Un estudio ${ }^{22}$ muestra que el uso del cloruro de diaquilcarbamilo tiene un efecto beneficioso en la curación de heridas, mientras que en este mismo estudio ${ }^{22}$ y en otro ${ }^{37}$ se indica que es eficaz en la cicatrización o disminución del tamaño de las heridas produciendo la mejoría de estas.

\section{UTILIZACIÓN DE LA SULFADIAZINA DE PLATA}

La sulfadiazina de plata muestra buenos resultados en la curación de heridas o en la progresión de estas ${ }^{34}$, aunque hay controversia sobre qué 

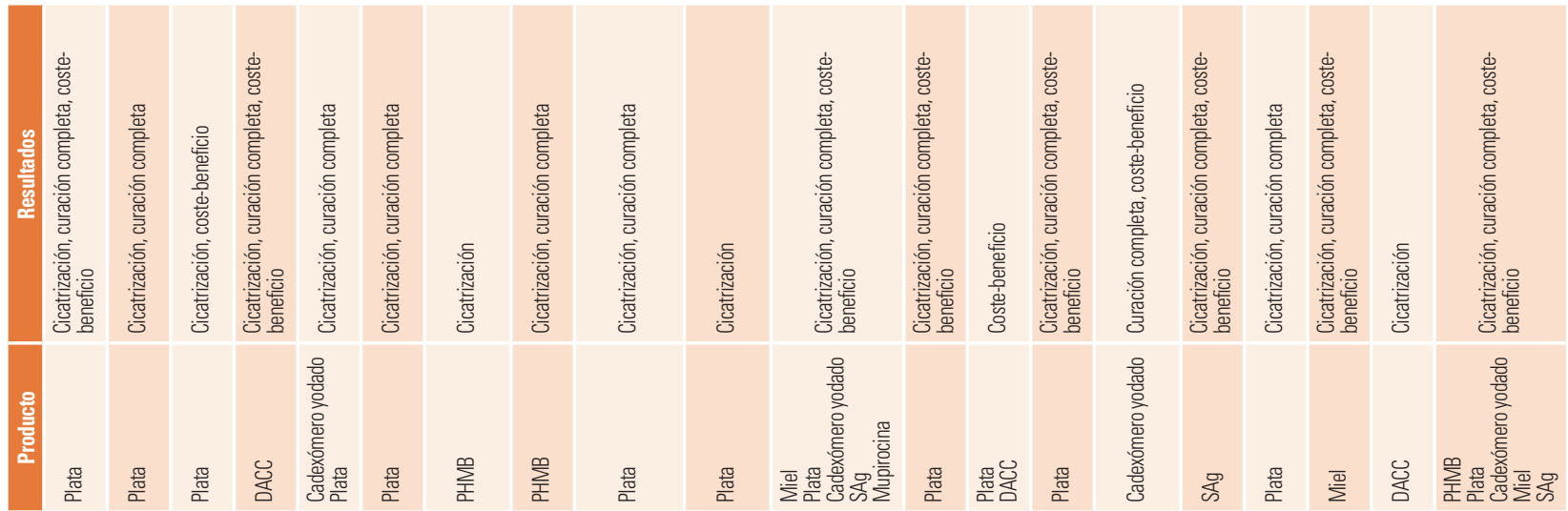

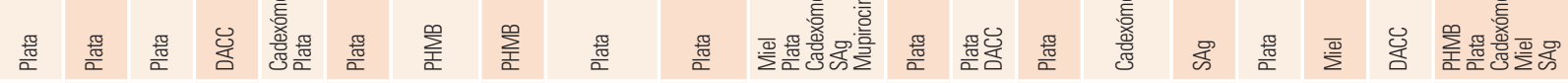
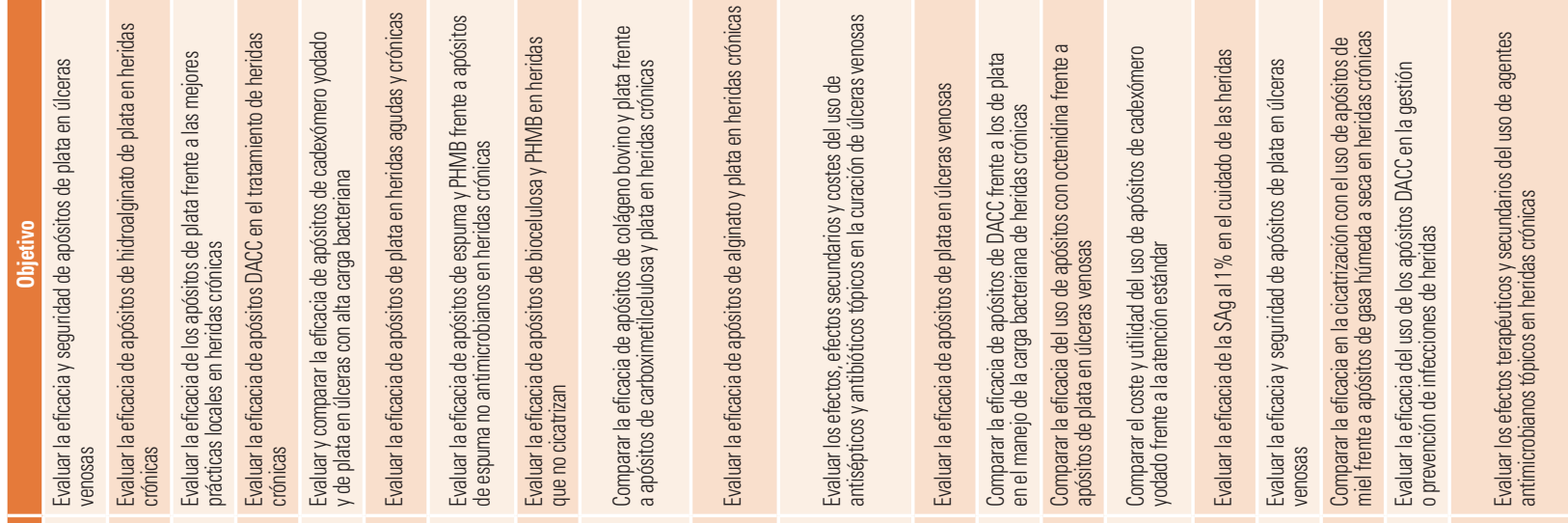

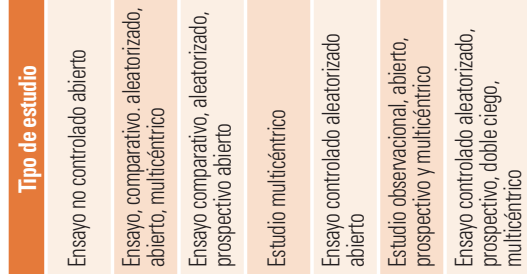
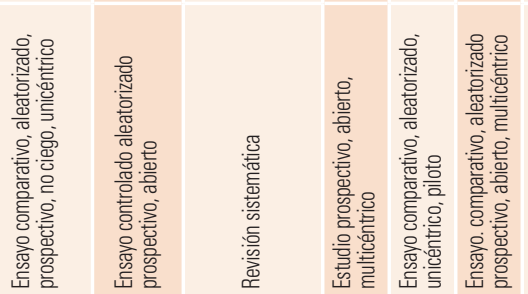

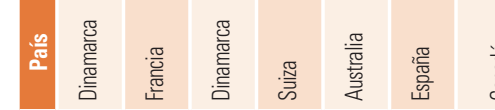

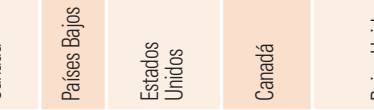
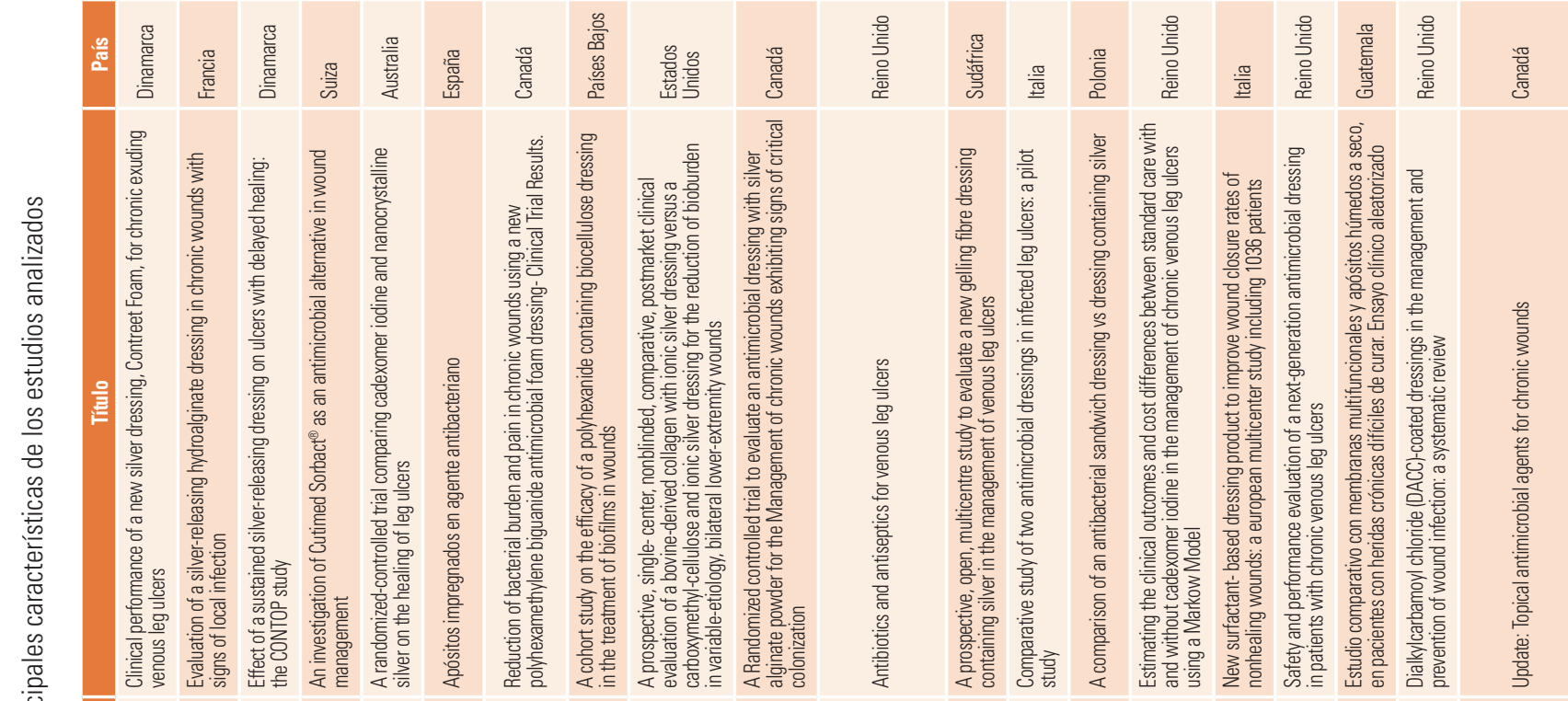

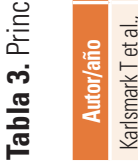

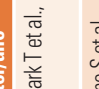
intions

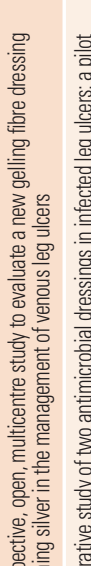

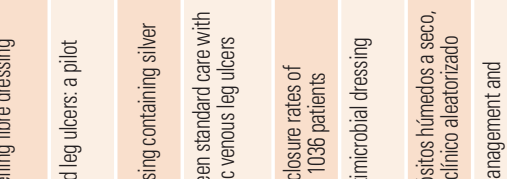

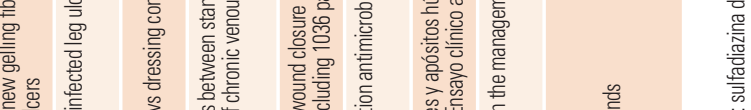

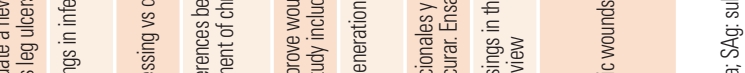

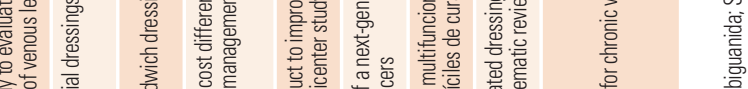

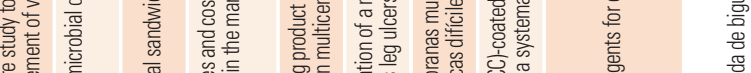

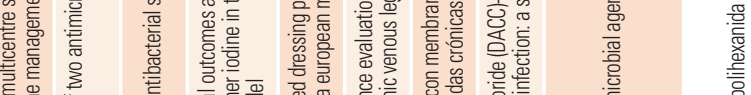

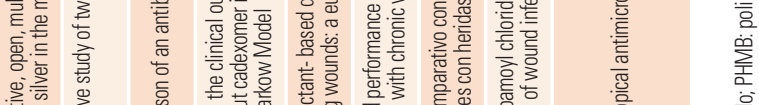

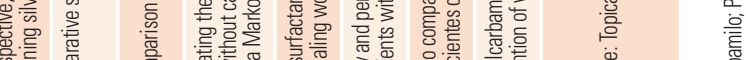

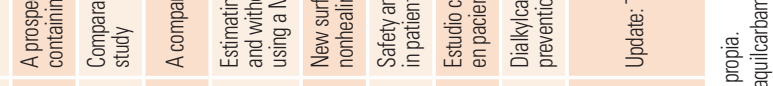

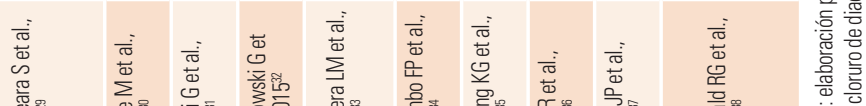

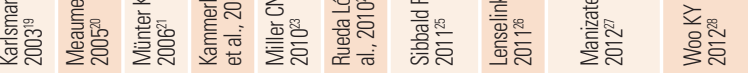


producto es más efectivo en la disminución del tamańo al compararla con la plata ${ }^{38}$.

En una revisión ${ }^{29}$ se obtienen resultados sobre la curación que son no significativos al comparar el uso de este producto con el cobre tripéptido, placebo o apósitos no adherentes.

\section{UTILIZACIÓN DE LA MUPIROCINA}

En un artículo ${ }^{29}$ se compara el número de pacientes que curaron su herida con el uso de la mupirocina frente al tul de parafina, pero los resultados son no significativos.

\section{Resultados sobre el coste-beneficio}

\section{UTILIZACIÓN DE LA PLATA}

En varios estudios ${ }^{30,31}$ en los que se utilizan apósitos de plata se obtiene el coste del tratamiento por paciente, resultando ser el más económico el Aquacel $\mathrm{Ag}^{31}$, aunque en este mismo estudio se compara con el cloruro de diaquilcarbamilo (Cutimed Sorbact), teniendo este producto un coste aún menor. A pesar de ello, es importante mencionar que la duración del tratamiento en cada estudio es diferente.

En otras investigaciones ${ }^{21,29,32,38}$ se compara el uso de la plata frente a otros productos, siendo esta más rentable que las mejores prácticas lo$\mathrm{cales}^{21}$, ya que se obtiene ahorro de tiempo de enfermería y de número de apósitos utilizados, coincidiendo dos estudios ${ }^{19,21}$ en el número de apósitos utilizados en cada paciente, aunque en uno de estos artícu$\operatorname{los}^{19}$ no se compara con ningún otro producto. También se obtiene un coste más asequible al compararla con la sulfadiazina de plata ${ }^{38}$.

Sin embargo, la plata no parece ser tan asequible en algunos de estos estudios $^{29,32}$ al compararla con otro apósito no antimicrobiano ${ }^{29}$ y con la octenidina ${ }^{32}$, debido a que la cantidad de apósitos utilizados es ligeramente superior y la reducción de la herida con cada apósito es menor al utilizar la plata frente a la octenidina ${ }^{32}$.

\section{UTILIZACIÓN DEL CADEXÓMERO YODADO}

El uso del cadexómero yodado es más rentable que los apósitos hidrocoloides $^{29}$ y la atención estándar ${ }^{33}$, produciéndose un notable ahorro económico y un mayor beneficio para el paciente, ya que en uno de estos estudios ${ }^{33}$, los pacientes que son tratados con cadexómero yodado junto con la atención estándar consiguen estar más tiempo libres de ulceración y una mayor puntuación QALY (años de vida ajustados por calidad) que al utilizar la atención estándar de manera exclusiva.

\section{UTILIZACIÓN DE LA MIEL}

La miel, por su parte, es poco rentable, ya que en los tratamientos con este producto se necesita un mayor gasto de apósitos ${ }^{36}$. Además, otro estudio $^{29}$ apoya la baja rentabilidad de la miel.

\section{UTILIZACIÓN DEL CLORURO DE DIAQUILCARBAMILO}

El cloruro de diaquilcarbamilo es una buena alternativa debido a su rentabilidad en el tratamiento de heridas crónicas infectadas o con signos de colonización crítica, como se muestra en dos artículos ${ }^{22,31}$, consiguiendo en uno de estos estudios ${ }^{31}$ un coste menor al compararlo con los apósitos de plata.

\section{UTILIZACIÓN DE LA SULFADIAZINA DE PLATA}

Por último, en un artículo ${ }^{34}$ se indica que con la aplicación de la sulfadiazina de plata se produce ahorro económico. Sin embargo, en otro estudio $^{38}$, el coste del tratamiento con este producto es notablemente superior al compararlo con la plata.

\section{У DISCUSIÓN}

Son muchos los posibles tratamientos existentes para las heridas crónicas infectadas o con signos de colonización crítica, existiendo en el mercado una amplia gama de productos.

\section{Eficacia de la plata}

El uso de la plata es sin duda el tratamiento más utilizado en heridas crónicas con signos de infección o con signos de colonización crítica.

A pesar de la variedad de productos existentes en el mercado, la plata en sus diferentes formas (nanocristalina, metálica e iónica) es la que se utiliza como primer escalón terapéutico en estas lesiones, como muestran numerosos artículos ${ }^{19-21,23,24,27,28-32,35,38}$, debido a los buenos resultados que se obtienen en la disminución del tamaño de las heridas o incluso en algunas ocasiones en la curación.

Sin embargo, existen algunos aspectos que juegan en su contra, como que no puede utilizarse junto con la colagenasa, ya que se inactiva al entrar en contacto con esta enzima, impidiendo el desbridamiento enzimático en caso de presencia de esfacelos o tejido necrótico.

Por otro lado, el coste y el beneficio obtenido en el paciente es considerablemente mejor que el de los demás tratamientos.

\section{Eficacia del cadexómero yodado}

El cadexómero yodado se utiliza como segundo escalón terapéutico, debido a que la plata obtiene mejores resultados que este producto en la disminución del tamaño de las heridas ${ }^{23}$.

Este producto presenta la ventaja de tener una liberación lenta y progresiva del yodo en la herida, a diferencia de otros productos yodados como la povidona yodada, siendo más rentable en este tipo de heridas que algunos apósitos de cura en ambiente húmedo ${ }^{29}$.

\section{Eficacia de la miel}

La miel es un producto natural que tiene excelentes propiedades antimicrobianas, estimulando así la curación de las heridas ${ }^{36}$, sobre todo la miel de Manuka.

Se presenta en forma de apósitos, siendo necesario que tenga el sello CE para que pueda ser considerada un producto sanitario y se pueda utilizar en las heridas, ya que sin este sello se trataría de miel alimentaria y no se garantiza la esterilidad y la seguridad del producto, lo que podría poner en riesgo la salud del paciente.

Sin embargo, este producto es poco rentable ${ }^{29}$, aunque es necesaria una mayor investigación.

\section{Eficacia de la polihexanida de biguanida}

La polihexanida de biguanida es un producto alternativo. A pesar de sus excelentes propiedades, no se utiliza tan frecuentemente en la práctica clínica como la plata o el cadexómero yodado, quizás debido a que la plata es la primera opción terapéutica y ambos productos no se pueden utilizar de forma conjunta. 


\section{Eficacia del cloruro de diaquilcarbamilo}

Este producto es efectivo en la curación ${ }^{22}$ y en la mejoría de las heridas $^{22,37}$, presentando ciertas ventajas en comparación con otros productos, por lo que debe considerarse como una buena alternativa a los demás tratamientos en el caso de que no sean efectivos o bien tolerados, ya que además es un producto bastante rentable ${ }^{22,31}$.

\section{Eficacia de la sulfadiazina de plata}

La sulfadiazina de plata debe de utilizarse como tercer escalón terapéutico, ya que se trata de un antibiótico tópico.

A pesar de potenciar la curación y/o cicatrización de las heridas ${ }^{34,38}$, el coste de su tratamiento es superior al de los apósitos de plata ${ }^{38}$.

\section{$\vee$ CONCLUSIONES}

- Existe una amplia gama de productos destinados al cuidado de las heridas crónicas infectadas o con signos de colonización crítica.
- La plata es el producto con el que se obtienen mejores resultados en la curación y/o disminución del tamaño de las heridas, siendo el primer escalón terapéutico.

- Los demás productos: el cadexómero yodado, la miel, la polihexanida de biguanida, el cloruro de diaquilcarbamilo y la sulfadiazina de plata, también obtienen buenos resultados en la curación y/o disminución del tamaño de las heridas, aunque hay menos estudios que los avalen, sobre todo en el caso de los antibióticos locales tópicos.

- La plata es el producto con mejor coste-beneficio, seguido del cadexómero yodado y la sulfadiazina de plata.

- El cloruro de diaquilcarbamilo puede ser una alternativa costo-efectiva al manejo de la carga bacteriana.

- Los tratamientos con miel son más caros, ya que se necesita un mayor gasto de apósitos. Además, uno de los estudios apoya que la miel es poco rentable.

\section{Conflicto de intereses}

Los autores declaran la no existencia de conflictos de interés relacionados con este artículo.

\section{У BIBLIOGRAFÍA}

1. Barón Burgos MM, Benítez Ramírez MM, Caparrós Cervantes A, Escarvajal López ME, Martín Espinosa MT, Moh Al-Lal Y, et al. Guía para la Prevención y Manejo de las UPP y heridas crónicas. Madrid: Instituto Nacional de Gestión Sanitaria; 2015

2. García Fernández FP, López Casanova P, Segovia Gómez T, Soldevilla Agreda JJ, Verdú Soriano J. Unidades Multidisciplinares de Heridas Crónicas: Clínicas de Heridas. Serie de Documentos de posicionamiento GNEAUPP n ${ }^{0}$ 10. 1. ${ }^{\text {a }}$ ed. Logroño: Grupo Nacional para el Estudio y Asesoramiento en Úlceras por Presión y Heridas Crónicas; 2012.

3. Ballesteros Úbeda MV. Heridas crónicas. En: Muñoz Rodríguez A, ed. Manual de Protocolos y Procedimientos en el cuidado de las heridas. Madrid: Hospital Universitario de Móstoles; 2011. p. 143-8.

4. García Fernández FP, Soldevilla Agreda JJ, Pancorbo Hidalgo PL, Verdú Soriano J, López Casanova P, Rodríguez Palma M. Clasificación-categorización de las lesiones relacionadas con la dependencia. Serie Documentos Técnicos GNEAUPP no II. 2. ${ }^{\text {a }}$ ed. Logroño: Grupo Nacional para el Estudio y Asesoramiento en Úlceras por Presión y Heridas Crónicas; 2014.

5. Allué Gracia MA, Ballabriga Escuer MS, Clerencia Sierra M, Gallego Domeque L, García Espot A, Moya Porté MT. Heridas crónicas: un abordaje integral. Huesca: Colegio Oficial de Enfermería de Huesca; 2012.

6. Ramírez Hernández GA. Fisiología de la cicatrización cutánea. RFS 2010;2(2):69-78.

7. Sainz-Espiga Michelena I, Folguera Álvarez C, Lebrancón Cortés P, Valerdiz Casasola S. Colonización crítica: la gran invisible. 1. ${ }^{\text {a ed. España: }}$ Coloplast Productos Médicos; 2016.

8. Carville K, Cuddigan J, Fletcher J, Fuchs P, Harding K, Ishikawa O, et al. La infección de las heridas en la práctica clínica. Consenso internacional. Londres: Medical Education Partnership Ltd; 2008.

9. García Fernández FP, Soldevilla Agreda JJ, Pancorbo Hidalgo PL, Verdú Soriano J, López Casanova P, Rodríguez Palma M, et al. Manejo Local de Úlceras y Heridas. Serie Documentos Técnicos GNEAUPP no III. 2. ed. Logroño: Grupo Nacional para el Estudio y Asesoramiento en Úlceras por Presión y Heridas Crónicas; 2018.

10. Pancorbo Hidalgo PL, Verdú Soriano J, Martínez Cuervo F. Manejo de la carga bacteriana. En: García Fernández FP, Soldevilla Agreda JJ, Torra i

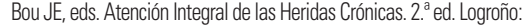
GNEAUPP-FSJJ; 2016. p. $461-75$

11. Pérez Santos L, Turrado Muñoz MÁ, Moral Arroyo JA. Recursos materiales para el cuidado de heridas crónicas y agudas. En: Pérez Santos L, ed. Consultoría de heridas crónicas y agudas. Córdoba: Hospital Universitario Reina Sofía. Servicio Andaluz de Salud. Consejería de Salud. Junta de Andalucía; 2017. p. 74-104.

12. Soldevilla Ágreda JJ, Torra i Bou JE, Posnett J, Verdú Soriano J, San Miguel L, Mayan Santos JM. Una aproximación al impacto del coste económico del tratamiento de las úlceras por presión en España. Gerokomos. 2007;18(4):201-10.
13. Cabello JB. Plantilla para ayudarte a entender un Ensayo Clínico. En: CASPe, ed. Guías CASPe de Lectura Crítica de la Literatura Médica. Alicante: CASPe; 2005. p. 5-8.

14. Cabello JB. Plantilla para ayudarte a entender una Revisión Sistemática. En: CASPe, ed. Guías CASPe de Lectura Crítica de la Literatura Médica. Alicante: CASPe; 2005. p. 13-7.

15. Cabello JB. Plantilla para ayudarte a entender Estudios de Cohortes. En: CASPe, ed. Guías CASPe de Lectura Crítica de la Literatura Médica. Alicante: CASPe; 2005. p. 23-7.

16. Cabello JB. Plantilla para ayudarte a entender una Evaluación Económica. En: CASPe, ed. Guías CASPe de Lectura Crítica de la Literatura Médica. Alicante: CASPe; 2005. p. 31-5.

17. Wang J, Smith J, Babidge W, Maddern G. Silver dressings versus other dressings for chronic wounds in a community care setting. J Wound Care. 2007;16(8):352-6.

18. Zößß C, Cech JD. Efficacy of a new multifunctional surfactant-based biomaterial dressing with $1 \%$ silver sulphadiazine in chronic wounds. Int Wound J. 2016;13(5):738-43.

19. Karlsmark T, Bendz SH, Larsen JR, Andersen KE, Roed-Petersen J, Agerslev RH. Clinical performance of a new silver dressing, Contreet Foam, for chronic exuding venous leg ulcers. J Wound Care. 2003;12(9):351-4.

20. Meaume S, Vallet D, Nguyen Morere M, Téot L. Evaluation of a silver-releasing hydroalginate dressing in chronic wounds with signs of local infection. J Wound Care. 2005;14(9):411-9.

21. Münter KC, Beele H, Russell L, Crespi A, Gröchenig E, Basse P, et al. Effect of a sustained silver-releasing dressing on ulcers with delayed healing: the CONTOP study. J Wound Care. 2006;15(5):199-206.

22. Kammerlander G, Locher E, Suess-Burghart A, von Hallern B, Wipplinger P. An investigation of Cutimed Sorbact $\circledast$ as an antimicrobial alternative in wound management. Wounds UK. 2008;4(2):10-4.

23. Miller CN, Newall N, Kapp SE, Lewin G, Karimi L, Carville K, et al. A randomized-controlled trial comparing cadexomer iodine and nanocrystalline silver on the healing of leg ulcers. Wound Repair Regen 2010;18(4):359-67.

24. Rueda López J, Martínez Cuervo F, Verdú Soriano J, Segovia Gómez T. Apósitos impregnados en agente antibacteriano. Rev ROL Enf. 2010;33(11):726-35

25. Sibbald RG, Coutts P, Woo KY. Reduction of Bacterial Burden and Pain in Chronic Wounds Using a New Polyhexamethylene Biguanide Antimicrobial Foam Dressing-Clinical Trial Results. Adv Skin Wound Care. 2011;24(2):78-84

26. Lenselink E, Andriessen A. A cohort study on the efficacy of a polyhexanide-containing biocellulose dressing in the treatment of biofilms in wounds. J Wound Care. 2011;20(11):534-9.

27. Manizate F, Fuller A, Gendics C, Lantis JC. A prospective, single-center, nonblinded, comparative, postmarket clinical eva- luation of a bovine-derived collagen with ionic silver dressing versus a carboxymethylcellulose and ionic silver dressing for the reduction of bioburden in variable-etiology. Adv Skin Wound Care. 2012;25(5):220-5.

28. Woo KY, Coutts PM, Sibbald RG. A randomized controlled trial to evaluate an antimicrobial dressing with silver alginate powder for the management of chronic wounds exhibiting signs of critical colonization. Adv Skin Wound Care. 2012;25(11):503-8.

29. O'Meara S, Al-Kurdi D, Martyn-St James M, Ovington LG, Richardson $\mathrm{R}$, Ologun Y. Antibiotics and antiseptics for venous leg ulcers. Cochrane Database Syst Rev. 2014;1:CD003557. doi: 10.1002/14651858. CD003557.pub5

30. Forlee M, Rossington A, Searle R. A prospective, open, multicentre study to evaluate a new gelling fibre dressing containing silver in the management of venous leg ulcers. Int Wound $J$. 2014:11(4):438-45

31. Mosti G, Magliaro A, Mattaliano V, Picerni P, Angelotti N. Comparative study of two antimicrobial dressings in infected leg ulcers: a pilot study. J Wound Care. 2015;24(3):121-7.

32. Krasowski G, Rybak Z, Bartoszewicz M, Olejniczak-Nowakowska M, Junka $A$, Tukiendorf $A$, et al. A comparison of an antibacterial sandwich dressing vs dressing containing silver. Wound Repair Regen. 2015;23(4):525-30.

33. Nherera LM, Woodmansey E, Trueman P, Gibbons GW. Estimating the Clinical Outcomes and Cost Differences Between Standard Care With and Without Cadexomer lodine in the Management of Chronic Venous Leg Ulcers Using a Markov Model. Ostomy Wound Manage. 2016;62(6):26-40.

34. Palumbo FP, Harding KG, Abbritti F, Bradbury S, Ivins N, Klein D, et al. New Surfactant-based Dressing Product to Improve Wound Closure Rates of Nonhealing Wounds:A European Multicenter Study Including 1036 Patients. Wounds. 2016;28(7):233-40.

35. Harding KG, Cains J, Blair S, Parsons D, Ivins NM, Bowler P, et al. Safety and performance evaluation of a next-generation antimicrobial dressing in patients with chronic venous leg ulcers. Int Wound $\mathrm{J}$. 2016;13(4):442-8.

36. Díaz R, Percy-Douglas R, Alvarado-Dumas C. Estudio comparativo con membranas multifuncionales y apósitos húmedos a seco, en pacientes con heridas crónicas difíiciles de curar. Ensayo clínico aleatorizado. Rev Guatem cir. 2017:23(1):24-35

37. Totty JP, Bua N, Smith GE, Harwood AE, Carradice D, Wallace T, et al. Dialkylcarbamoyl chloride (DACC)-coated dressings in the management and prevention of wound infection: a systematic review. J Wound Care. 2017:26(3):107-14.

38. Sibbald RG, ElliottJA, Verma L, Brandon A, Persaud R, Ayello EA. Update: Topical Antimicrobial Agents for Chronic Wounds. Adv Skin Wound Care. 2017;30(10):438-50. 